\title{
PERMANENT GENETIC RESOURCES NOTE Permanent Genetic Resources added to Molecular Ecology Resources Database 1 February 2013-31 March 2013
}

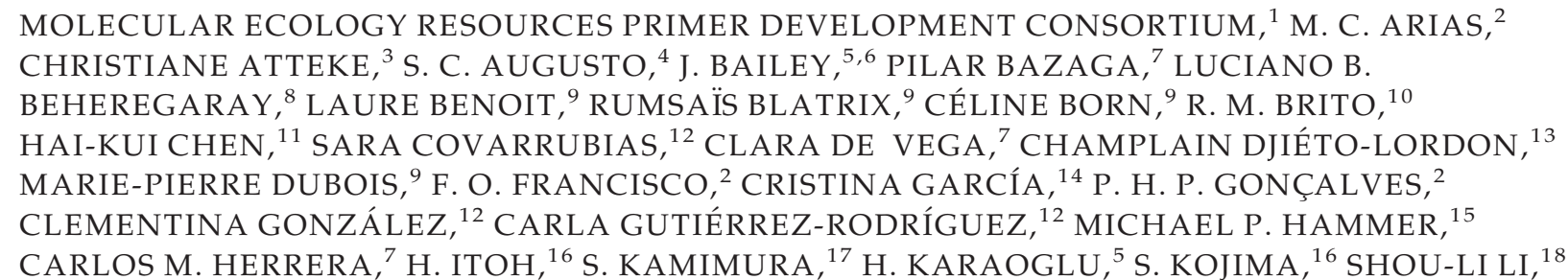
HANNAH J. LING ${ }^{8}$ PÁVEL F. MATOS-MARAVÍ, ${ }^{19,20}$ DOYLE MCKEY, ${ }^{9,}{ }^{, 21}$ JUDICAËL MEZUI-M ${ }^{\prime}$ EKO,${ }^{3}$ JUAN FRANCISCO ORNELAS, ${ }^{12}$ R. F. PARK,${ }^{5}$ MARÍA I. POZO,${ }^{7}$ SATU RAMULA, ${ }^{18,22,23}$ CRISTINA RIGUEIRO, ${ }^{24}$ JONATHAN SANDOVAL-CASTILLO ${ }^{8}$ L. R. SANTIAGO,${ }^{2}$ MIYUKI M. SEINO, ${ }^{7}$ CHANG-BING SONG,${ }^{11}$ H. TAKESHIMA, ${ }^{16}$ ANTI VASEMÄGI, ${ }^{19,25}$ C. R. WELLINGS, ${ }^{5}$ JI YAN, ${ }^{26}$ DU YU-ZHOU, ${ }^{26}{ }^{C H A N G-R O N G ~ Z H A N G ~}{ }^{27}$ and TIAN-YUN ZHANG ${ }^{28}$

${ }^{1}$ Molecular Ecology Resources Editorial Office, 6270 University Blvd, Vancouver, British Columbia Canada, V6T 1Z4, ${ }^{2}$ Departamento de Genética e Biologia Evolutiva, Instituto de Biociências, Universidade de São Paulo, Rua do Matão, 277, São Paulo, SP 05508-090, Brazil, ${ }^{3}$ Département de Biologie, Faculté des Sciences, Université des Sciences et Techniques de Masuku (USTM), B.P. 901, Franceville, Gabon, 'Instituto de Biologia, Universidade Federal de Uberlândia, Rua Ceará S/N, Bloco 2D, Campus Umuarama, Uberlândia, MG 38400-902, Brazil, ${ }^{5}$ Faculty of Agriculture and Environment, Plant Breeding Institute, The University of Sydney, 107 Cobbitty Rd, Cobbitty, NSW 2570, Australia, ${ }^{6}$ Cooperative Research Centre for National Plant Biosecurity, LPO Box 5012, Bruce, ACT 2617, Australia, ${ }^{7}$ Estación Biológica de Doñana, CSIC, Avda. Américo Vespucio s/n, Sevilla, 41092, Spain, ${ }^{8}$ Molecular Ecology Laboratory, School of Biological Sciences, Flinders University, Adelaide, SA 5001, Australia, ${ }^{9}$ Centre d'Ecologie Fonctionnelle et Evolutive (CEFE), UMR 5175 (CNRS, Université Montpellier 2, CIRAD-Bios), 1919 route de Mende, 34293 Montpellier cedex 5, France, ${ }^{10}$ Instituto de Genética e Bioquimica, Universidade Federal de Uberlândia, Rua Pará 1720, Bloco 2E, Campus Umuarama, Uberlândia, MG 38400-902, Brazil, ${ }^{11}$ Department of Life Science, Beifang University of Nationalities, Yinchuan 730000, China, ${ }^{12}$ Departamento de Biología Evolutiva, Instituto de Ecología, A.C., Carretera Antigua a Coatepec 351, El Haya, Xalapa, Veracruz 91070, Mexico, ${ }^{13}$ Laboratory of Zoology, Faculty of Science, University of Yaoundé I, PO Box 812, Yaoundé, Cameroon, ${ }^{14}$ Research Center in Biodiversity and Genetic Resources (CIBIO), Campus Agrário de Vairão, Rua Padre Armando Quintas, Vairão 4485-661, Portugal, ${ }^{15}$ Museum and Art Gallery of the Northern Territory, PO Box 4646, Darwin, NT 0810, Australia, ${ }^{16}$ Atmosphere and Ocean Research Institute, The University Of Tokyo, 5-1-5, Kashiwanoha, Kashiwa, Chiba 277-8564, Japan, ${ }^{17}$ National Institute For Land and Infrastructure Management, Ministry Of Land, Infrastructure and Transport, 3-1-1, Nagase, Yokosuka, Kanagawa 239-0826, Japan, ${ }^{18}$ Section of Ecology, Department of Biology, University of Turku, 20014 Turku, Finland, ${ }^{19}$ Division of Genetics and Physiology, Department of Biology, University of Turku, 20014, Turku, Finland, ${ }^{20}$ Biology Center AS CR, School of Biological Sciences, University of South Bohemia and Institute of Entomology, Branisovska 31, 37005 Ceske Budejovice, Czech Republic, ${ }^{21}$ Institut Universitaire de France, ${ }^{22}$ Biodiversity Unit, Department of Biology, Lund University, 22362 Lund, Sweden, ${ }^{23}$ Aronia Coastal Zone Research Team, Åbo Akademi University, Raseborgsvägen 9, 10600 Ekenäs, Finland, ${ }^{24}$ Integrative Ecology Group, Estación Biológica de Doñana, Consejo Superior de Investigaciones Científicas (EBD-CSIC), Avenida Americo Vespucio s/n, E-41092 Sevilla, Spain, ${ }^{25}$ Department of Aquaculture, Institute of Veterinary Medicine and Animal Science, Estonian University of Life Sciences, 51014 Tartu, Estonia, ${ }^{26}$ College of Horticulture and Plant Protection \& Institute of Applied Entomology, Yangzhou University, Yangzhou, Jiangsu 225009, China, ${ }^{27}$ Department of Lanzhou Military Area Command, Institute of Drug and Instruments Control of Joint Logistics, Lanzhou 730050, China, ${ }^{28}$ The First Hospital, The Chinese People's Liberation Army (PLA), Lanzhou, 730050, China 


\begin{abstract}
This article documents the addition of 142 microsatellite marker loci to the Molecular Ecology Resources database. Loci were developed for the following species: Agriophyllum squarrosum, Amazilia cyanocephala, Batillaria attramentaria, Fungal strain CTeY1 (Ascomycota), Gadopsis marmoratus, Juniperus phoenicea subsp. turbinata, Liriomyza sativae, Lupinus polyphyllus, Metschnikowia reukaufii, Puccinia striiformis and Xylocopa grisescens. These loci were cross-tested on the following species: Amazilia beryllina, Amazilia candida, Amazilia rutila, Amazilia tzacatl, Amazilia violiceps, Amazilia yucatanensis, Campylopterus curvipennis, Cynanthus sordidus, Hylocharis leucotis, Juniperus brevifolia, Juniperus cedrus, Juniperus osteosperma, Juniperus oxycedrus, Juniperus thurifera, Liriomyza bryoniae, Liriomyza chinensis, Liriomyza huidobrensis and Liriomyza trifolii.
\end{abstract}

This article documents the addition of 142 microsatellite marker loci to the Molecular Ecology Resources database. Table 1 contains information on the focal species, the number of loci developed, any other species the loci were tested in and the accession numbers for the loci in both the Molecular Ecology Resources database and GenBank.
The authors responsible for each set of loci are listed in the final column. A full description of the development protocol for the loci presented here can be found on the Molecular Ecology Resources database (http://tomato. biol.trinity.edu/).

Table 1 Information on the focal species, the number of loci developed, any other species the loci were tested in and the accession numbers for the loci in both the Molecular Ecology Resources database and GenBank. The authors responsible for each set of loci are listed in the final column

\begin{tabular}{|c|c|c|c|c|c|}
\hline Species & $\begin{array}{l}\text { No. primers } \\
\text { developed }\end{array}$ & $\begin{array}{l}\text { Other species } \\
\text { tested }\end{array}$ & MER database no. & GenBank accession no. & Authors \\
\hline $\begin{array}{l}\text { Agriophyllum } \\
\text { squarrosum }\end{array}$ & 15 & $\mathrm{n} / \mathrm{a}$ & $50753-50767$ & JX477772-JX477786 & $\begin{array}{l}\text { Zhang, Tian-Yun; } \\
\text { Zhang, Chang-Rong; } \\
\text { Song, Chang-Bing; } \\
\text { Chen, Hai-kui }\end{array}$ \\
\hline $\begin{array}{l}\text { Amazilia } \\
\text { cyanocephala }\end{array}$ & 10 & $\begin{array}{l}\text { A. beryllina, } \\
\text { A. candida, } \\
\text { A. rutila, } \\
\text { A. tzacatl, } \\
\text { A. violiceps, } \\
\text { A. yucatanensis, } \\
\text { Campylopterus } \\
\text { curvipennis, } \\
\text { Cynanthus } \\
\text { sordidus, } \\
\text { Hylocharis } \\
\text { leucotis }\end{array}$ & $50666-50675$ & $\begin{array}{c}\text { КС566118, KC566120, } \\
\text { КС566121, КС566123, } \\
\text { КС566125-КС566130 }\end{array}$ & $\begin{array}{l}\text { Gutiérrez-Rodríguez, } \\
\text { Carla; } \\
\text { Covarrubias, Sara; } \\
\text { González, Clementina; } \\
\text { Ornelas, Juan } \\
\text { Francisco }\end{array}$ \\
\hline $\begin{array}{l}\text { Batillaria } \\
\text { attramentaria }\end{array}$ & 14 & $\mathrm{n} / \mathrm{a}$ & $50686-50699$ & $\begin{array}{l}\text { AB711161, } \\
\text { AB711163-AB711166, } \\
\text { AB762682-AB762690 }\end{array}$ & $\begin{array}{l}\text { Itoh, H.; } \\
\text { Kamimura, S.; } \\
\text { Takeshima, H.; } \\
\text { Kojima, S. }\end{array}$ \\
\hline $\begin{array}{l}\text { Fungal } \\
\text { strain CTeY1 } \\
\text { (Ascomycota) }\end{array}$ & 9 & $\mathrm{n} / \mathrm{a}$ & $50647-50655$ & КС551930-КС551938 & $\begin{array}{l}\text { Benoit, Laure; } \\
\text { Blatrix, Rumsaï; } \\
\text { Djiéto-Lordon, } \\
\text { Champlain; } \\
\text { Atteke, Christiane; } \\
\text { Mezui-M'Eko, } \\
\text { Judicaël; } \\
\text { Dubois, Marie-Pierre; } \\
\text { McKey, Doyle; } \\
\text { Born, Céline }\end{array}$ \\
\hline
\end{tabular}


Table 1 (Continued)

\begin{tabular}{|c|c|c|c|c|c|}
\hline Species & $\begin{array}{l}\text { No. primers } \\
\text { developed }\end{array}$ & $\begin{array}{l}\text { Other species } \\
\text { tested }\end{array}$ & MER database no. & GenBank accession no. & Authors \\
\hline $\begin{array}{l}\text { Gadopsis } \\
\text { marmoratus }\end{array}$ & 13 & $\mathrm{n} / \mathrm{a}$ & $50725-50737$ & КС662062-КС662074 & $\begin{array}{l}\text { Ling, Hannah J.; } \\
\text { Sandoval-Castillo, } \\
\text { Jonathan; } \\
\text { Hammer, Michael P.; } \\
\text { Beheregaray, } \\
\text { Luciano B. }\end{array}$ \\
\hline $\begin{array}{l}\text { Juniperus } \\
\text { phoenicea } \\
\text { subsp. turbinata }\end{array}$ & 10 & $\begin{array}{l}\text { J. brevifolia, } \\
\text { J. cedrus, } \\
\text { J. osteosperma, } \\
\text { J. oxycedrus, } \\
\text { J. thurifera }\end{array}$ & $50676-50685$ & КС538839-КС538848 & $\begin{array}{l}\text { García, Cristina; } \\
\text { Rigueiro, Cristina }\end{array}$ \\
\hline $\begin{array}{l}\text { Liriomyza } \\
\text { sativae }\end{array}$ & 10 & $\begin{array}{l}\text { L. bryoniae, } \\
\text { L. chinensis, } \\
\text { L. huidobrensis, } \\
\text { L. trifolii }\end{array}$ & $50656-50665$ & $\begin{array}{l}\text { JX988506, JX988507, } \\
\text { JX988510, } \\
\text { JX988511, JX988513, } \\
\text { JX988515-JX988519 }\end{array}$ & $\begin{array}{l}\text { Yan, Ji; } \\
\text { Yu-Zhou, Du }\end{array}$ \\
\hline $\begin{array}{l}\text { Lupinus } \\
\text { polyphyllus }\end{array}$ & 12 & $\mathrm{n} / \mathrm{a}$ & $50738-50749$ & КС $814577-$ КС 814588 & $\begin{array}{l}\text { Li, Shou-Li; } \\
\text { Vasemägi, Anti; } \\
\text { Matos-Maraví, } \\
\text { Pável F.; } \\
\text { Ramula, Satu }\end{array}$ \\
\hline $\begin{array}{l}\text { Metschnikowia } \\
\text { reukaufii }\end{array}$ & 11 & $\mathrm{n} / \mathrm{a}$ & $50768-50778$ & КС802230-KC802240 & $\begin{array}{l}\text { Seino, Miyuki M.; } \\
\text { de Vega, Clara; } \\
\text { Bazaga, Pilar; } \\
\text { Pozo, María I.; } \\
\text { Herrera, Carlos M. }\end{array}$ \\
\hline $\begin{array}{l}\text { Puccinia } \\
\text { striiformis }\end{array}$ & 25 & $\mathrm{n} / \mathrm{a}$ & $50700-50724$ & $\begin{array}{l}\text { AEEW01006106, AEEW01006924, } \\
\text { AEEW01007191, AEEW01007248, } \\
\text { AEEW01007581, AEEW01007642, } \\
\text { AEEW01007821, AEEW01008284, } \\
\text { AEEW01008708, AEEW01008827, } \\
\text { AEEW01008886, AEEW01009186, } \\
\text { AEEW01009408, AEEW01009492, } \\
\text { AEEW01009750, AEEW01009975, } \\
\text { AEEW01010273, AEEW01010833, } \\
\text { AEEW01011699, AEEW01012705, } \\
\text { AEEW01012792, AEEW01014724, } \\
\text { AEEW01014764, AEEW01027613, } \\
\text { AEEW01028302 }\end{array}$ & $\begin{array}{l}\text { Bailey, J.; } \\
\text { Karaoglu, H.; } \\
\text { Wellings, C. R.; } \\
\text { Park, R. F. }\end{array}$ \\
\hline $\begin{array}{l}\text { Xylocopa } \\
\text { grisescens }\end{array}$ & 13 & $\mathrm{n} / \mathrm{a}$ & $50634-50646$ & КC168056-КC168068 & $\begin{array}{l}\text { Augusto, S. C.; } \\
\text { Gonçalves, P. H. P.; } \\
\text { Brito, R. M.; } \\
\text { Francisco, F. O.; } \\
\text { Santiago, L. R.; } \\
\text { Arias, M. C. }\end{array}$ \\
\hline
\end{tabular}

